\title{
Encoding LED for Unique Markers on Object Recognition System
}

\author{
Wildan Pandji Tresna ${ }^{1}$, Umar Ali Ahmad ${ }^{2}$, Isnaeni ${ }^{3}$, Reza Rendian Septiawan ${ }^{4}$, Iyon Titok Sugiarto ${ }^{5}$, Alex Lukmanto \\ Suherman ${ }^{6}$ \\ Research Center for Physics, National Research and Innovation Agency, Banten, Indonesia ${ }^{1,3,5,6}$ \\ Department of Computer Engineering, School of Electrical Engineering, Telkom University, Bandung, Indonesia ${ }^{1,2,4}$
}

\begin{abstract}
In this paper a new approach of unique markers to detect and track moving objects with the encoding LED marker is presented. In addition, an LED spotlight system that can shoot light in the direction of the target is also proposed. The encoding process is done by making a unique blinking pattern on the LED, and thus the camera and a servomotor as an object recognition system would only recognize a unique marker given by an LED. In this work the camera with OpenCV could capture a unique marker in all variant of blinking patterns. A unique marker is important on object recognition system so the camera could identify the object marked by our unique marker and ignore all other markers that might captured by the camera. In addition, analysis of the PWM signal from an LED is carried out here to determine the characteristics of the LEDs in each color, determine the accuracy of the duty cycle, and the use of the bright-dim method on the LEDs. The results show that the highest accuracy is obtained when a $50 \%$ duty cycle is used with the on and off time are set to be 1 second for all LED colors. The benefit of the proposed system is confirmed by implementing an integrated control system as a unique marker. The effectiveness of the blinking LED against other laser interferences is also discussed.
\end{abstract}

Keywords-Encoding LED; PWM signal; target markers; object recognition system

\section{INTRODUCTION}

The optics and optoelectronics have made significant progress over the years, particularly towards more powerful and efficient laser devices [1]. Much of these advances are responsible for making them indispensable in modern warfare. There has been a large-scale growth of electro-optics devices and systems for various applications, such as target tracking and designating, range finding, and target acquisition [2][3]. Moreover, [4] and [5] suggested that the use of lasers for military purposes continues to grow every year. There are numerous benefits associated with electro-optics technology, for example, detecting markers by using a light-emitting diode (LED) [6][7].

LED has some benefits, such as low power consumption and more brightness compared to the others [8]. LED technology also presents an option for information display on a flat screen, offering a wide viewing angle and a bright and clear image suitable for outdoor applications. LEDs have become the necessary option in lighting environmental renovation. LEDs have many advantages, including high fidelity, high rendering, and supporting green technology [9][10]. In [11] it is reported that LED pulse response behavior, taking into account the junction capacitance and the spreading resistance distributed over the entire junction area, this research makes possible to be able to control LED in the future. LED has recently become a new light source in many areas due to its efficiency and durability. It has the merits of being environmentally friendly and low power consumption. Furthermore, LEDs are used in various sensors and other technological developments, such as LED markers [12][13].

Recently, LEDs cannot only emit continuously, but also dim and blinking. The author in [14] reported the dimming is accomplished by adjusting the average current in the LEDs through pulse width modulation of a switch in series with the load. Meanwhile, [15] suggested that a camera with image processing software is used to recognize the target. The color, size, and variant of blinking of the LED markers detected by the camera is the main limiting factor of this method.

In [16] it is reported that the object recognition system by using single camera. The quality of the camera greatly influences the object detection limits. Meanwhile, the increase of interest and need for the camera generates increasingly complex object tracking algorithms. The object tracking system is often used for security, surveillance, traffic monitoring, navigation, and human-computer interactions [17][18]. Moreover, LED marker is a marker of a particular light. This marker is usually used just before the bullet is fired.

LED needs the electric current to emit the light. The higher the current flowing in the LED, the brighter the light produced. However, it should be noted that the amount of current allowed is $10 \mathrm{~mA}-20 \mathrm{~mA}$ and a voltage of $1.6 \mathrm{~V}-3.5 \mathrm{~V}$ according to the character of the wavelength. If the current is higher than 20mA, the LED will burn. Changing the electric current on LED according to a certain pattern at a certain time can produce LED blinking [19][20]. In this work, the aim to develop an object recognition system and unique marker by using a variety of LED blinking. The LED spotlight and object recognition based on computer vision were studied, resulting in the fact that the correlation was small.

\section{METHOD}

\section{A. Scheme of LED Blinking}

The diode forward current can control the LED luminance. The most convenient method for LED dimming without altering the current is the pulse-width-modulation (PWM). PWM regulates the output voltage on the LED to get different average voltages. Meanwhile, PWM adjusts the output voltage 
signal on the led to get different average voltages. Two factors limiting the shortest PWM pulse duration are driver response time and LED response time. Typically, driver response time is clearly stated by a manufacturer. LED manufacturers are not aware of the need for faster LED response times. Usually, the manufacturer considers it to be fast enough to satisfy dimming requirements. However, the LED manufacturers do not specify the response time. Therefore, the investigation was needed to evaluate the current state of the response time of visible light LEDs.

In this work, LED is controlled by a microcontroller, which can show the continuous or blinking light, as shown in Fig. 1. Here, the LED is controlled by an Arduino Uno and will generate a PWM. In addition, the Arduino Uno can adjust the blinking signal with a variety of frequencies and duty cycles. For the optimum condition, the maximum speed of the duty cycle on LED depends on the speed rate of spectrometer capture led light.

The spectrometer used in this work has a limitation of speed rate in $10 \mu \mathrm{s}$. Therefore, the speed of the duty cycle was set at over $10 \mu \mathrm{s}$. The spectrometer has captured the spectrum of LED and converted it to the PWM signal. The comparison between the PWM signal in the input and the output was analyzed to generate the precision and resolution numbers.

\section{B. Object Recognition System}

The detection of objects using markers is one of the easiest methods of detection. Markers are artificial objects that are designed to be easily recognized and identified. The marker usually is at a known position or size, serving as a reference point or a measure for other objects. In this work, a camera with image processing software called OpenCV is used to recognize the target. The motor servo is used to steer the laser beam. An external camera is used to recognize the target. This camera can capture pictures with a resolution of $1920 \times 1080$ pixels for $30 \mathrm{fps}$ and a $76^{\circ}$ angle of view. However, the camera also needs a particular program to recognize the object intended as the target. There are several methods of OpenCV that can be used for target recognition. Since the target is dynamic (moving objects), the shape and size of the target, which is recognized by the camera, might slightly change when it moves.
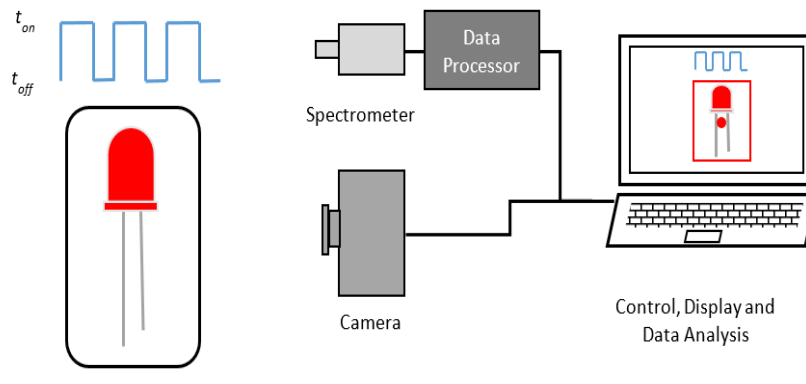

LED

Fig. 1. Encoding LED Blinking Mechanism. A Camera Captured the Display of LED, and a Spectrometer Captured the PWM Signal.

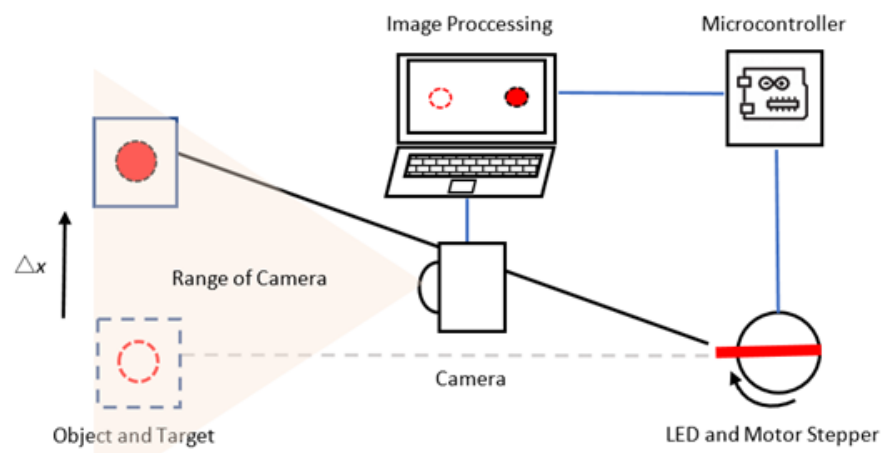

Fig. 2. Object Recognition System consists of the Camera, Image Processing, LED as a Marker Embedded to the Servo Motor and Microcontroller.

The object recognition system consists of multi-colored LED that can emit continuous light, external cameras for capturing an object, and a spectrometer for capturing a PWM signal. When the LED was on, the camera captured the visualization of the colored LED, and the spectrometer captured the PWM signal simultaneously as shown in Fig. 2. Visually, the light looks on and off with the particular period. The PWM signal goes up $\left(t_{o n}\right)$ and down $\left(t_{\text {off }}\right)$ on a particular blinking type, and the duty cycle follows the rules as shown in (1). When the light is on, $t_{o n}$ is always straight without breaking up, and there is no signal at the $t_{\text {off, vice versa. The }}$ blinking system is the combination of both $t_{\text {on }}$ and $t_{\text {off }}$ that can produce the PWM signal. Typically, the camera will capture the visual object when the light is on $\left(t_{o n}\right)$ and will be lost when the light is off $\left(t_{o f f}\right)$.

\section{RESULT AND DiSCUSSION}

\section{A. Characteristics of LED Blinking}

In this work, LEDs of various colors have been characterized and analyzed for their resolution and precision values. The colors used in this experiment are red, yellow, green, and blue. The duty cycle is the fraction of one period when a signal is active, and its calculation follows (1). Moreover, the PWM duty cycle's accuracy is related to the duty cycle's correction. The precision analysis of Duty Cycle (DC) follows (2).

$D C=\frac{t_{\text {on }}}{t_{\text {on }}+t_{\text {off }}} \times 100 \%$

Accuracy of PWM DC $=\left[1-\left(\frac{D C_{\text {inp }}-D C_{\text {out }}}{D C_{\text {inp }}}\right)\right] \times 100 \%$

Here, the duty cycle is the ratio of time a load, and the $t_{\text {on }}$ and $t_{\text {off }}$ are the conditions when the light is on and off. The duty cycle correction for the color LED can be calculated by comparing the input duty cycle and the output duty cycle. The four colors have different wavelengths, resulting in different accuracies. It can be seen on the $t_{\text {on }}$ and $t_{\text {off, where the time }}$ variations are carried out to see the maximum speed captured by the photo detector and spectrum processing software. The concept of $t_{d i m}$ was proposed to capture all the time on Computer Vision as shown in Fig. 3. 


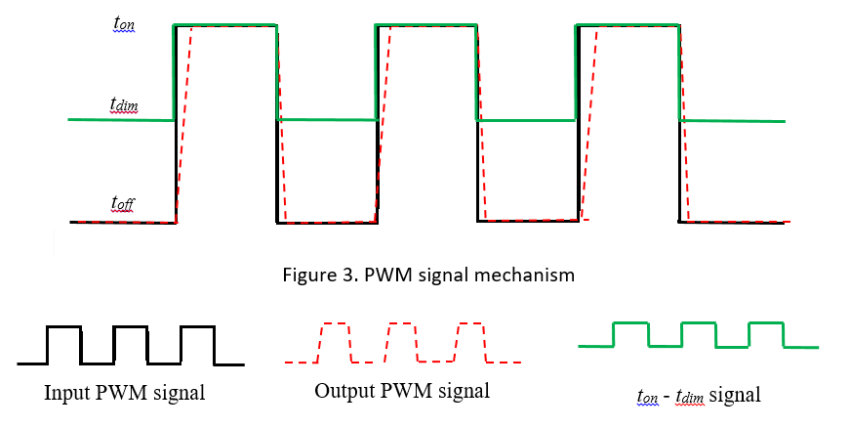

Fig. 3. The Mechanism of PWM Signal, separately by the Various Color.

Initially, the LED has a $50 \%$ duty cycle. It means that in the blinking system, the length of time when it turns on is the same as the length of time it turns off. Following this, the PWM signal was analyzed. In this work, the duty cycle is calculated by $t_{o n}=1 \mathrm{~s}$ and $t_{o f f}=1 \mathrm{~s}$. Furthermore, the variety of duty cycles and range of time were analyzed as well.

When the duty cycle is gradually decreased, the accuracy of the duty cycle decreases, as shown in Fig. 4a. The smaller the duty cycle, the $t_{\text {off }}$ is higher than a $t_{o n}$, the duty cycle's accuracy drops significantly. Due to the photo detector's response, these phenomena are also affected by the speed ratio $\left(t_{o n}\right.$ and $\left.t_{o f f}\right)$. Meanwhile, the duty cycle data after $50 \%$ show no significant accuracy shift, as shown in Fig. 4b. Therefore, $50 \%$ duty cycle is a simpler form of PWM signal and gives the higher accuracy of the duty cycle. The result indicates that when the LED switched from on to off, the spectrometer still receives the LED beam signal in a short time. The increasing value of the duty cycle correlates with the decrease's correction value. The result shows that at a longer $t_{o n}$, the photo detector will capture the object better. The combination of $t_{o n}$ and $t_{o f f}$, correction values, and accuracy of duty cycle describes the encoding system for object recognition systems.
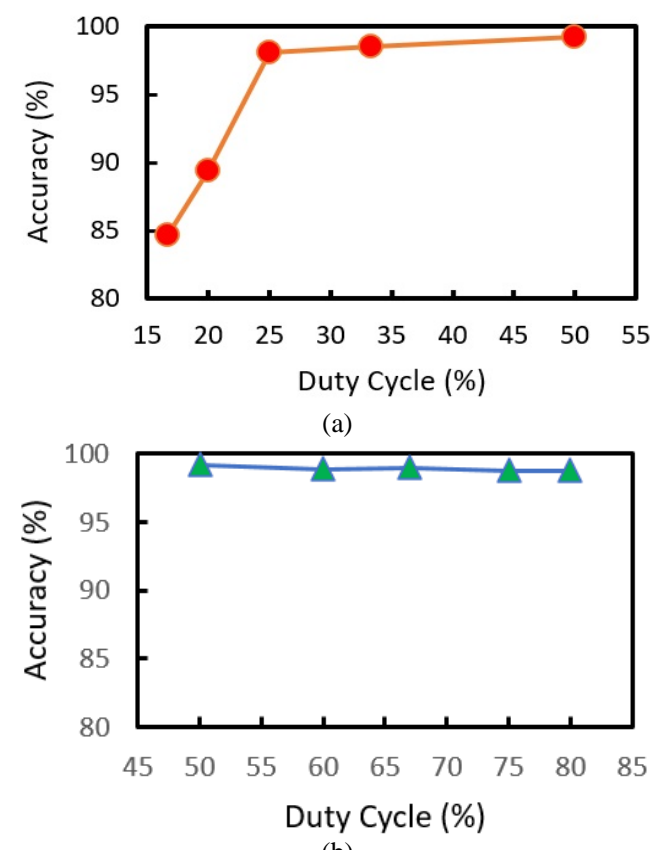

(b)

Fig. 4. Duty Cycle Dependency of Accuracy on the Red LED.

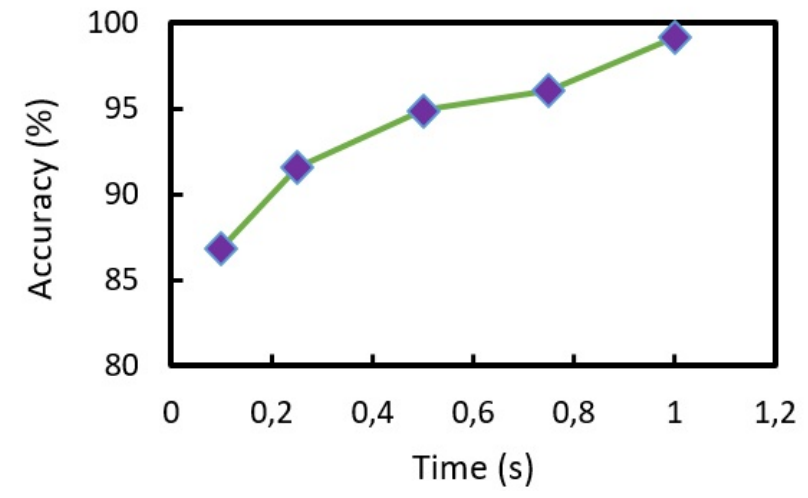

Fig. 5. The $t_{o n}$ and $t_{o f f}$ Dependency of Accuracy on the Red LED under $1 \mathrm{~s}$.

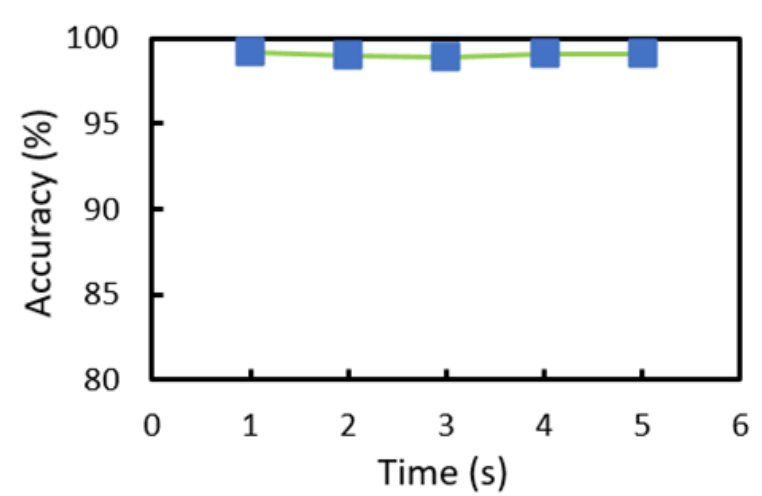

Fig. 6. The $t_{o n}$ and $t_{\text {off }}$ Dependency of Accuracy on the Red LED after $1 \mathrm{~s}$.

On the data of the $50 \%$ duty cycle, the time dependencies of accuracy were analyzed. When the $t_{o n}$ and $t_{\text {off }}$ are getting smaller, the blinking speed increases, and the accuracy of the duty cycle drops significantly, as shown in Fig. 5. Meanwhile, the variant $t_{o n}$ and $t_{\text {off }}$ data after one second with the periodic time indicates no significant accuracy shift, as shown in Fig. 6.

Therefore, one second of $t_{o n}$ and $t_{\text {off }}$ is the cut-off point of time signal and gives the higher accuracy of the duty cycle. Here, all the measurement is on the operating range of the spectrometer. The transformation of the accuracy of the duty cycle due to the variant of $t_{o n}$ and $t_{\text {off }}$ describes the blinking system. Due to the photo detector's response, these phenomena are also affected by the speed ratio $\left(t_{\text {on }}\right.$ and $\left.t_{\text {off }}\right)$.
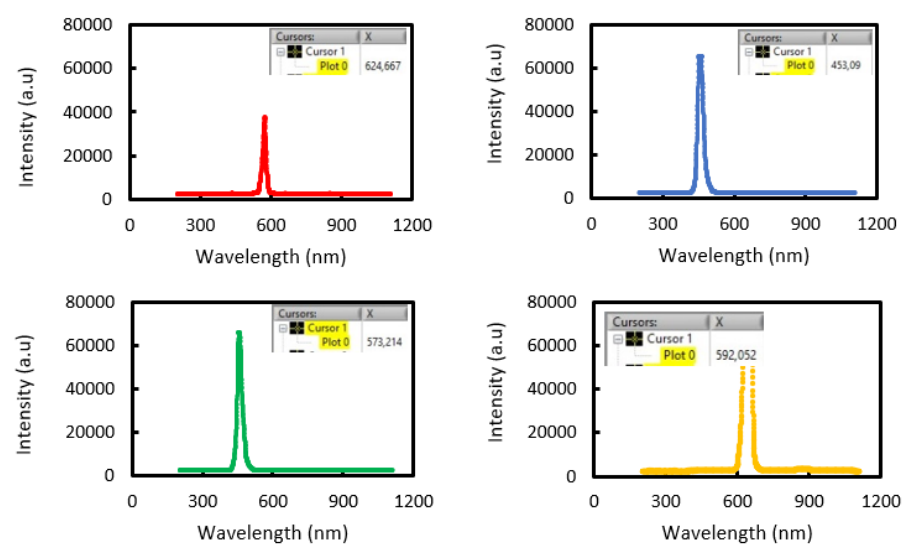

Fig. 7. The Various Wavelength on the Red, Blue, Green and Yellow LED. 

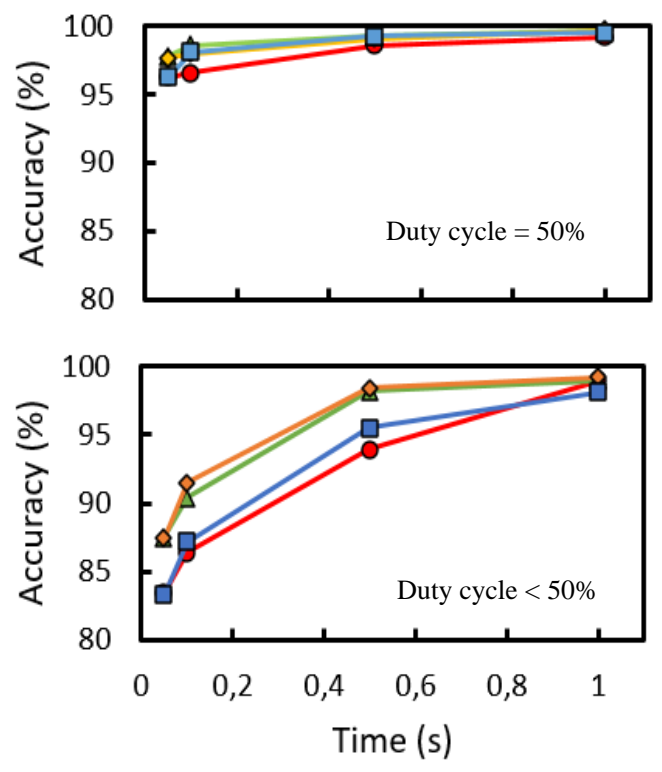

Fig. 8. Time Dependency of Precision Value on the Several Wavelengths LED.

The enhanced measurement was also analyzed with the same method based on the experimental result of duty cycle and variant of $t_{\text {on }}$ and $t_{\text {off. }}$ Fig. 7 shows that the red LED has $624.67 \mathrm{~nm}$ in wavelength. Meanwhile, blue, green, and yellow have $453.09 \mathrm{~nm}, 573.21$, and 592.05, respectively.

On the $50 \%$ duty cycle data, the time dependencies of accuracy were analyzed on the various color of the LED, as shown in Fig. 8 (upper side). When the $t_{\text {on }}$ and $t_{\text {off }}$ are getting shorter, almost the color of LED has lower accuracy, especially at the $t_{\text {on }}=t_{\text {off }}=0.05 \mathrm{~s}$ and $0.1 \mathrm{~s}$. When the variant of $t_{\text {on }}$ and $t_{\text {off }}$ is higher, all the colors of LED get closer to similar accuracy. This result shows LEDs with the various wavelengths has similar accuracy at the highest duty cycle. Moreover, the shortest time range produces the smallest accuracy when the duty cycle is lower than $50 \%$, as shown in Fig. 8 (lower side). Meanwhile, the duty cycle after $50 \%$ shows the similar accuracy of duty cycle. The time dependencies accuracy increases significantly when the time range increases periodically. On the $t_{o n}$ and $t_{\text {off }}$ equal $1 \mathrm{~s}$, almost the variant of LED has a similar accuracy. Note that the red and blue LED has the lowest accuracy in almost all conditions.

\section{B. Object Recognition of LED Blinking}

LED with continuous light always keeps and locks on the computer vision as shown in Fig. 8. However, when the LED blinking is on, the LED is on $\mathrm{t}_{\text {off }}$, and the camera cannot recognize the object as shown in Fig. 9. In this work, the report evidence that the combination of $t_{\text {on }}$ and $t_{\text {off }}$ and the value of the duty cycle can lead computer vision always to capture objects and lock them in the visual display.

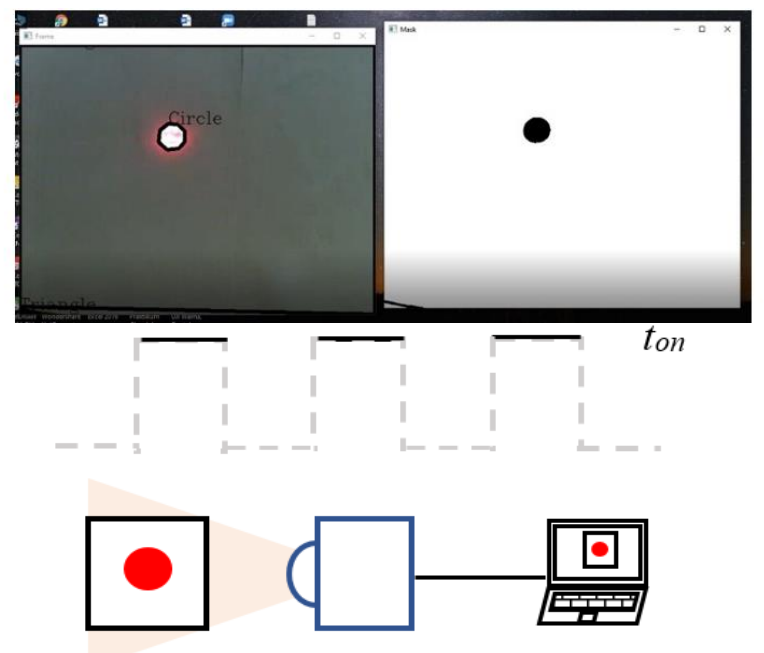

Fig. 9. The $t_{\text {on }}$ Condition, the Marker Captured Well by the Camera.

The display in Fig. 9 consists of the original frame and masking the frame. The original frame shows the object and the bounding of object contour for the object recognition system. Then the masking frame display is the original frame with the reducer noise filter. The masking frame is presented in the black and white mode. The object is represented in the white color, and all the background will show in black color area. In this condition, the PWM signal is always in upper position $\left(t_{o n}\right)$. However, $t_{o n}$ condition of LED in range of camera was captured continuously as sown in Fig. 9.

Meanwhile, Fig. 10 shows the original and the masking frame cannot recognize the object contour for $t_{\text {off }}$ condition. In this case, the PWM signal is always in under position $\left(t_{o n}\right)$. However, $t_{\text {off }}$ condition of LED in range of camera was not captured continuously. Here, the dotted circle is the representative of the LED position.

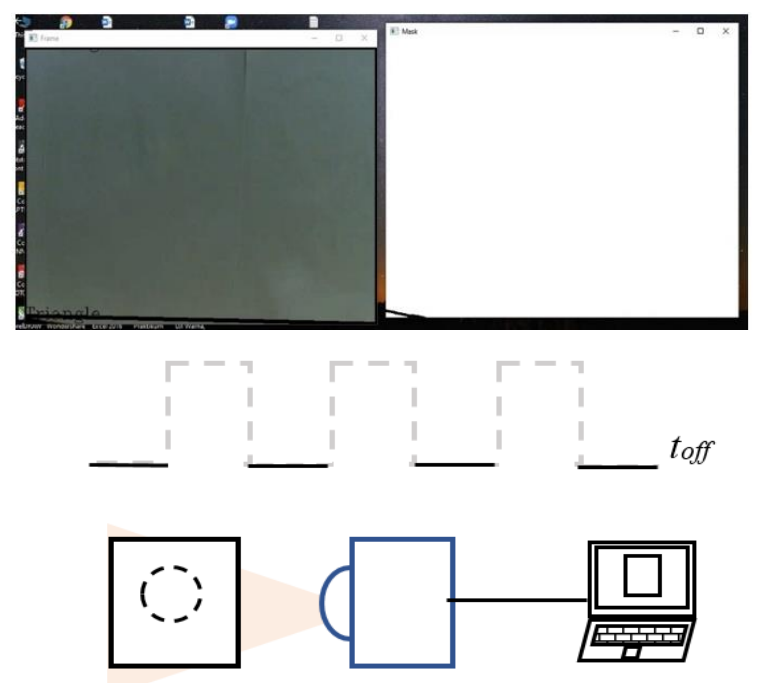

Fig. 10. In the $t_{\text {off }}$ Condition, the Marker is not Captured All the Time by the Camera. 

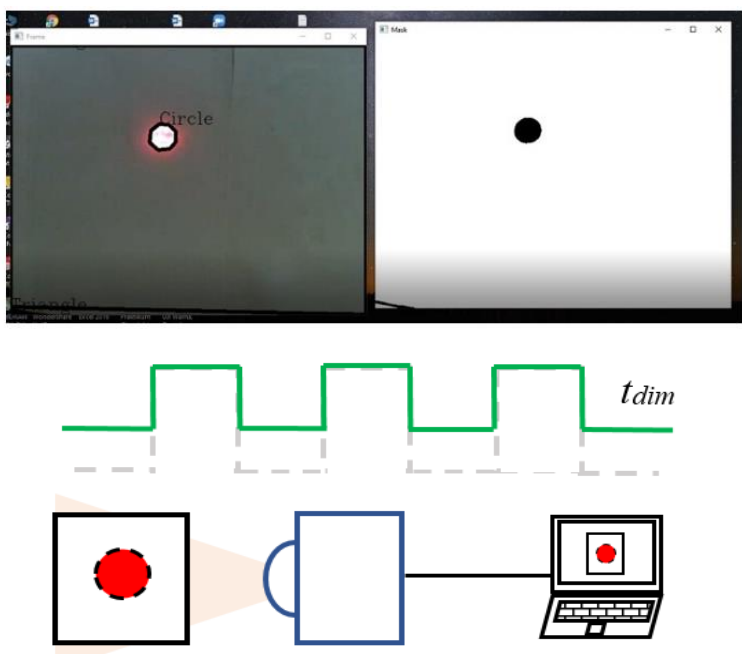

Fig. 11. The Combination between $t_{o n}$ and $t_{d i m}$, the Marker Captured Well by the Camera.

The unique blinking of encoding LED as a marker is captured perfectly on the camera as well as shown in Fig. 11. As long as the unique blinking consists of the bright-dim method, a camera always locks the $x$-axis and $y$-axis. However, the bright-dim condition was proposed to solve the $t_{\text {off }}$ condition as shown in Fig. 10. In bright-dim condition, the PWM signal is always in upper and middle position. However, $t_{\text {bright }}-t_{\text {dim }}$ condition of LED in range of camera was captured continuously. LED with bright-dim condition always keeps and locks on the computer vision as shown in Fig. 11.

Fig. 12 shows the unique blinking concept and the evident of it sent in other files. This unique marker will be recognized, and the distractor markers will not be recognized by camera.

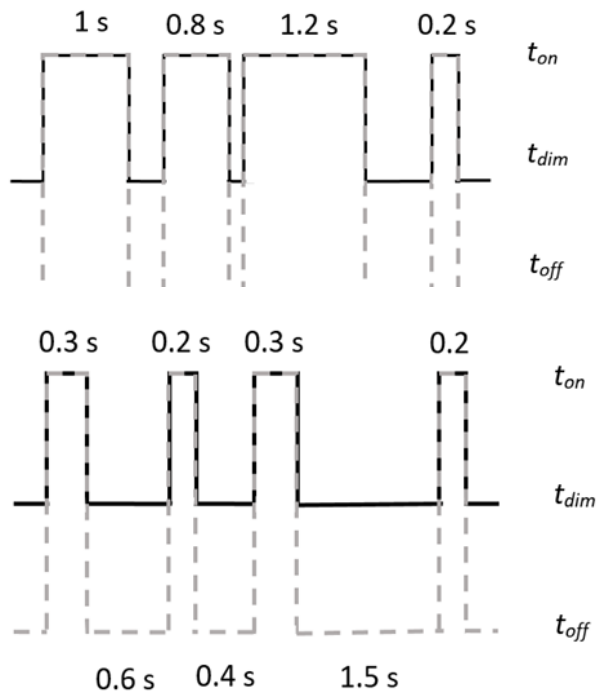

Fig. 12. The Variant of PWM Signals that can be produced to Unique Markers. A Combination of Bright-Dim Captures the Markers All the Time on a Camera.

\section{CONCLUSION}

A new object recognition approach with the unique LED marker is studied rapidly to propose an encoding marker. Camera and image processing would only recognize the unique marker. Characterization LED blinking has been successfully analyzed by controlling the PWM signal with a variety of duty cycles. The accuracy of the duty cycle is one of the parameters to describe the characteristics of LED blinking. The experiment results show that the highest accuracy is obtained on a $50 \%$ duty cycle with the range of time $1 \mathrm{~s}$ on any LED color. The object recognition system captured all variant LED blinking with the bright-dim method.

This method potentially applied for the future work to the smart weapon in the military system, especially the movement of three-dimensional objects that can applied to air-ground smart missiles.

\section{ACKNOWLEDGMENT}

The Author's would like to thank all of member LASER group, Research Center for Physics, National Research and Innovation Agency for the entire instrument that used in this work.

\section{REFERENCES}

[1] B. G. Disha and G. Indumathi, "Automated test system for laser designator and laser range finder," IJERT, vol. 4, no. 5, pp. 126-130, 2015.

[2] L. Goldberg, J. Nettleton, B. Schilling, W. Trussel, and A. Hays, "Compact laser sources for laser designation, ranging and active imaging," in Proc. SPIE 6552, Orlando, Florida, USA, 2007, pp. 65520G1-65520G8.

[3] H. Kaushal and G. Kaddoum, "Application of laser and tactical military operatins," IEEE Access, vol. 5 pp. 20736-20753, 2017.

[4] L. Lazov, E. Teirumnieks, and R. S. Ghalot, "Applications of laser technology in the army," J. Def. Manag, vol. 11 Iss. 4 No: 210. 2018.

[5] M. L. W. B. Anderberg, "Laser weapons: The dawn of a new military age," New York, USA: Springer, 2013.

[6] A. K. Maini, "Battlefield lasers and opto-electronics systems," Def. Sci. Journal, vol. 60, no. 2, pp. 189-196. 2010.

[7] P.H. Putman, "When old is new again," Video Systems pp. 37-43, 2002.

[8] E.F. Schubert, "Light-emitting diodes," Cambridge University Press, 2003, p. 313.

[9] G. Leschhorn and R. Young, "Handbook of led and ssl metrology, instrumentation system," GmbH, 2017.

[10] H. I Hsieh, H. W. "LED current balance using a variable voltage regulator with low dropout ds control," Applied Sciences, pp. 1-14. 2017.

[11] I. Hino and K. Iwamoto. "LED pulse response analysis considering the distributed CR constant in the peripheral junction," IEEE Trans. on Elec. Dev. Vol. 26, pp. 1238-1242. 1979.

[12] W. Kurdthongmee, and T. Lamsub. "An automatic system for nonuniform brightness compensation of LED arrays: image processing routines to locate LED centers," Walailak J. Sci \& Tech. vol. 5, no. 2, pp. 203-216. 2008.

[13] L. Svilainis. "LED PWM dimming linearity investigation. displays," vol. 29, pp. 243-249, 2008.

[14] P. Nara, and D. S. Zinger. "An effective LED dimming approach," in IAS Annual Meeting - IEEE Industry Applications Society, vol. 3, pp. 1671-1676, November 2004.

[15] K. Adi, A. P. Widodo, C. E. Widodo, A. Pamungkas, and A. B. Putranto, "Automatic vehicle counting using background subtraction method on gray scale images and morphology operation," in OP Conf. Series: J. of Physics: Conf. Series 1025, 012025, 2018. 
[16] R. T. Yunardi, A. W. Mardhiyah, M. H. Yahya, and F. C. S. Arisgraha, "Desain dan implementasi visual object tracking menggunakan Pan and Tilt vision system," ELKHA, vol. 11, no. 2, pp. 85 - 92, 2019.

[17] B. Micusik, and T. Pajdla, "Simultaneous surveillance camera calibration and foot-head homology estimation from human detections," In 2010 IEEE Computer Society Conference on Computer Vision and Pattern Recognition, pp. 1562-1569, 2010.

[18] H. G. Schantz, “A real-time location system using near-field electromagnetic ranging," 2007 IEEE Antennas and Propagation Society
International Symposium, Honolulu, Hawaii, USA, pp. 3792-3795, 2007.

[19] J. Biswas, and M. Veloso, "Depth camera based indoor mobile robot localization and navigation. In 2012 IEEE International Conference on Robotics and Automation, pp. 1697-1702, 2012.

[20] H. Adinanta, H. Kato, A. W. S. Putra, and T. Maruyama, "Enhancement of beam tracking response using color filtering method for optical wireless power transmission," AIP Conference Proceedings 2256, vol. 1, no. 6, 2020. 